\title{
Chaos Suppression and Stabilization of Generalized Liu Chaotic Control System
}

\author{
Yeong-Jeu Sun ${ }^{1}$, Jer-Guang Hsieh ${ }^{2}$ \\ ${ }^{1}$ Professor, ${ }^{2}$ Chair Professor
}

Department of Electrical Engineering, I-Shou University, Kaohsiung, Taiwan

\begin{abstract}
In this paper, the concept of generalized stabilization for nonlinear systems is introduced and the stabilization of the generalized Liu chaotic control system is explored. Based on the time-domain approach with differential inequalities, a suitable control is presented such that the generalized stabilization for a class of Liu chaotic system can be achieved. Meanwhile, not only the guaranteed exponential convergence rate can be arbitrarily prespecified but also the critical time can be correctly estimated. Finally, some numerical simulations are given to demonstrate the feasibility and effectiveness of the obtained results.
\end{abstract}

Key Words: Generalized synchronization, Liu chaotic system, critical time, exponential convergence rate

\section{INTRODUCTION}

In recent years, chaotic dynamic systems have been widely investigated by researchers; see, for instance, [1-12] and the references therein. Very often, chaos in many dynamic systems is an origin of the generation of oscillation and an origin of instability. For a chaotic control system, it is important to design a controller that has both good transient and steady-state response. Furthermore, suppressing the occurrence of chaos plays an important role in the controller design of a nonlinear system.

In the past decades, various methodologies in control design of chaotic system have been presented, such as variable structure control approach, time-domain approach, adaptive control approach, adaptive sliding mode control approach, back stepping control approach, and others.

In this paper, the concept of generalized stabilizability for nonlinear dynamic systems is introduced and the stabilizability of generalized Liu chaotic control system will be investigated. Based on the timedomain approach with differential inequality, a suitable control will be offered such that the generalized stabilization can be achieved for a class of Liu chaotic system. Not only the critical time can be correctly estimated, but also the guaranteed exponential convergence rate can be arbitrarily prespecified. Several numerical simulations will also be provided to illustrate the use of the main results.

The layout of the rest of this paper is organized as follows. The problem formulation, main result, and controller design procedure are presented in Section 2. Numerical simulations are given in Section 3 to show the effectiveness of the developed results. Finally, conclusion remarks are drawn in Section 4.

\section{PROBLEM FORMULATION AND MAIN RESULTS \\ Nomenclature \\ $\mathfrak{R}^{n} \quad$ the $n$-dimensional real space \\ $|a|$ the modulus of a complex number $a$ \\ $A^{T} \quad$ the transport of the matrix $A$ \\ $\|x\| \quad$ the Euclidean norm of the vector $x \in \mathfrak{R}^{n}$}

In this paper, we explore the following generalized Liu chaotic system:

$$
\begin{aligned}
\dot{x}_{1}(t)= & a_{1} x_{1}(t)+a_{2} x_{2}(t)+u_{1}(t), \\
\dot{x}_{2}(t)= & a_{3} x_{1}(t)+a_{4} x_{1}(t) x_{3}(t)+u_{2}(t), \\
\dot{x}_{3}(t)= & a_{5} x_{1}(t)+a_{6} x_{2}(t)+a_{7} x_{3}(t) \\
& +a_{8} x_{1}^{2}(t)+u_{3}(t), \quad \forall t \geq 0,
\end{aligned}
$$




$$
\begin{aligned}
& {\left[\begin{array}{lll}
x_{1}(0) & x_{2}(0) & x_{3}(0)
\end{array}\right]^{T}} \\
& =\left[\begin{array}{lll}
x_{10} & x_{20} & x_{30}
\end{array}\right]^{T},
\end{aligned}
$$

where $x(t):=\left[\begin{array}{lll}x_{1}(t) & x_{2}(t) \quad x_{3}(t)\end{array}\right]^{T} \in \mathfrak{R}^{3}$ is the state vector, $u(t):=\left[u_{1}(t) \quad u_{2}(t) \quad u_{3}(t)\right]^{T} \in \mathfrak{R}^{3}$ is the system control, $\left[\begin{array}{lll}x_{10} & x_{20} & x_{30}\end{array}\right]^{T}$ is the initial value, and $a_{i}, b \in \Re$ indicate the parameters of the system. The original Liu chaotic system is a special case of system (1) with $u(t)=0$, $a_{1}=-a_{2}=10, a_{3}=40, a_{4}=-1, a_{5}=a_{6}=0, a_{7}=-2.5$, and $a_{8}=4$. It is well known that the system (1) without any control (i.e., $u(t)=0$ ) displays chaotic behavior for certain values of the parameters [1]. The aim of this paper is to search a novel control for the system (1) such that the generalized stability of the feedbackcontrolled system can be guaranteed. In this paper, the concept of generalized stabilization will be introduced. Motivated by time-domain approach with differential inequality, a suitable control strategy will be established. Our goal is to design a control such that the generalized stabilization of system (1) can be achieved.

Let us introduce a definition which will be used in subsequent main results.

1 There exist two positive numbers $k$ and $b$, such that $\|x(t)\| \leq k \cdot e^{-b t}, \quad \forall t \geq 0$.

2 There exists a positive number $t_{c}$ such that $x(t)=0, \quad \forall t \geq t_{c}$.

\section{Definition 1}

The system (1) is said to realize the generalized stabilization, provided that there exist a suitable control $u$ such that the conditons (i) and (ii) are satisfied. In this case, the positive number $b$ is called the exponential convergence rate and the positive number $t_{c}$ is called the critical time.

Now we present the main result for the generalized stabilization of the system (1) via time-domain approach with differential inequalities.

\section{Theorem 1}

The system (1) realizes the generalized stabilization under the following control

$$
\begin{aligned}
u_{1}(t)= & -\left(a_{1}+b\right) x_{1}(t)-a_{2} x_{2}(t)-a x_{1}^{2 \alpha-1}(t),(2 \mathrm{a}) \\
u_{2}(t)= & -a_{3} x_{1}(t)-a_{4} x_{1}(t) x_{3}(t)-b x_{2}(t) \\
& -a x_{2}^{2 \alpha-1}(t), \\
u_{3}(t)= & -a_{5} x_{1}(t)-a_{6} x_{2}(t)-\left(a_{7}+b\right) x_{3}(t) \\
& -a_{8} x_{1}^{2}(t)-a x_{3}^{2 \alpha-1}(t),
\end{aligned}
$$

where $a>0, b>0, \alpha:=\frac{p+q-1}{2 p-1}$, with $p, q \in N$ and $p>q$

In this case, the pre-specified exponential convergence rate and the guaranteed critical time are given by $b$ and

$$
t_{c}=\frac{\ln \left[\frac{\frac{a}{b}}{\left[x_{1}^{2}(0)+x_{2}^{2}(0)+x_{3}^{2}(0)\right]^{1-\alpha}+\frac{a}{b}}\right]}{-2(1-\alpha) b},
$$

respectively

Proof. From (1)-(2), the feedback-controlled system can be performed

$$
\begin{aligned}
& \dot{x}_{1}=-b x_{1}-a\left(x_{1}\right)^{2 \alpha-1}, \\
& \dot{x}_{2}=-b x_{2}-a\left(x_{2}\right)^{2 \alpha-1},
\end{aligned}
$$$$
\dot{x}_{3}=-b x_{3}-a\left(x_{3}\right)^{2 \alpha-1} \text {, }
$$

Let

$W(x(t))=x^{T}(t) x(t)$.

The time derivative of $W(x(t))$ along the trajectories of feedback-controlled system is given by

$$
\begin{aligned}
\dot{W} & =2 x_{1} \cdot \dot{x}_{1}+2 x_{2} \cdot \dot{x}_{2} \\
& =-2 b \cdot x_{1}^{2}-2 b \cdot x_{2}^{2}-2 a \cdot x_{1}^{2 \alpha}-2 a \cdot x_{2}^{2 \alpha} \\
& =-2 b \cdot W-2 a\left(x_{1}^{2 \alpha}+x_{2}^{2 \alpha}\right) \\
& \leq-2 b \cdot W-2 a \cdot W^{\alpha}, \quad \forall t \geq 0 .
\end{aligned}
$$

It follows that

$(1-\alpha) W^{-\alpha} \dot{W}+2(1-\alpha) b W^{1-\alpha}$

$\leq-2 a(1-\alpha), \forall t \geq 0$.

Define

$Q(t):=W(x(t))^{1-\alpha}, \quad \forall t \geq 0$.

From (6) and (7), it can be readily obtained that $\dot{Q}+2(1-\alpha) b Q \leq-2 a(1-\alpha), \quad \forall t \geq 0$.

It is easy to deduce that

$e^{2(1-\alpha) b t} \cdot \dot{Q}(t)+e^{2(1-\alpha) b t} \cdot 2(1-\alpha) b Q(t)$

$=\frac{d}{d t}\left[e^{2(1-\alpha) b t} \cdot Q(t)\right]$

$\leq-2 a(1-\alpha) e^{2(1-\alpha) b t}, \quad \forall t \geq 0$.

It follows that

$\int_{0}^{t} \frac{d}{d t}\left[e^{2(1-\alpha) b t} \cdot Q(t)\right] d t$

$=e^{2(1-\alpha) b t} \cdot Q(t)-Q(0)$ 
$\leq \int_{0}^{t}-2 a(1-\alpha) e^{2(1-\alpha) b t} d t$

$=\frac{-a}{b}\left(e^{2(1-\alpha) b t}-1\right), \quad \forall t \geq 0$.

Consequently, we have

$Q(t) \leq\left[Q(0)+\frac{a}{b}\right] \cdot e^{-2(1-\alpha) b t}-\frac{a}{b}$,

$\forall t \geq 0$.

Hence, from (6), (7), and (8), we have

(i) If $0 \leq t \leq t_{c}$,

$W(x(t)) \leq\left[\left(\|x(0)\|^{2-2 \alpha}+\frac{a}{b}\right) \cdot e^{-2(1-\alpha) b t}-\frac{a}{b}\right]^{1 /(1-\alpha)} ;$ (ii) If $t \geq t_{c}$,

$x(t)=0$.

Consquently, we conclude that

(i) If $0 \leq t \leq t_{c}$,

$\|x(t)\| \leq\left[\|x(0)\|^{2-2 \alpha}+\frac{a}{b}\right]^{1 /(2-2 \alpha)}$

(ii) If $t \geq t_{c}, x(t)=0$,

in view of (5) with above condition (i). This completes the proof.

\section{NUMERICAL SIMULATIONS}

Consider the generalized Liu chaotic system of (1) with $a_{1}=-a_{2}=10, a_{3}=40, a_{4}=-1, a_{5}=a_{6}=0, a_{7}=-2.5$ , $a_{8}=4$, and $x(0)=\left[\begin{array}{lll}4 & 2 & -2\end{array}\right]^{T}$. Our objective, in this example, is to design a feedback control such that the system (1) realize the generalized stabilization with with the guaranteed exponential convergence rate $b=0.5$. From (2), with $a=100, p=3, q=2$, we deduce $\alpha=0.8$,

$u_{1}(t)=-10.5 x_{1}(t)+10 x_{2}(t)-100 x_{1}^{0.6}(t),(9 \mathrm{a})$

$u_{2}(t)=-40 x_{1}(t)+x_{1}(t) x_{3}(t)-0.5 x_{2}(t)$ $-100 x_{2}^{0.6}(t)$,

$u_{3}(t)=2 x_{3}(t)-4 x_{1}^{2}(t)-100 x_{3}^{0.6}(t)$,

Consequently, by Theorem 1, we conclude that the system (1) achieves generalized stabilization with parameters of $a_{1}=-a_{2}=10, a_{3}=40, a_{4}=-1, a_{5}=a_{6}=0$, $a_{7}=-2.5, a_{8}=4$, and feedback control law of (9). Furthermore, the exponential convergence rate and the guaranteed critical time are given by $b=0.5$ and $t_{c}=0.047$.

The typical state trajectories of uncontrolled systems and controlled systems are depicted in Figure 1 and Figure 2, respectively. From the foregoing simulations results, it is seen that the dynamic system of (1) achieves the generalized stabilization under the control law of (9).

\section{CONCLUSION}

In this paper, the concept of generalized stabilization for nonlinear systems has been introduced and the stabilization of generalized Liu chaotic control system has been studied. Based on the time-domain approach with differential inequalities, a suitable control has been presented such that the generalized stabilization for a class of Liu chaotic system can be achieved. Besides, not only the guaranteed exponential convergence rate can be arbitrarily pre-specified but also the critical time can be correctly estimated. Finally, some numerical simulations have been offered to show the feasibility and effectiveness of the obtained results.

\section{ACKNOWLEDGEMENT}

The authors thank the Ministry of Science and Technology of Republic of China for supporting this work under grants MOST 106-2221-E-214-007, MOST 106-2813-C-214-025-E, and MOST 107-2221E-214-030.

\section{REFERENCES}

1. D. Su, W. Bao, J. Liu, and C. Gong, "An efficient simulation of the fractional chaotic system and its synchronization," Journal of the Franklin Institute, vol. 355, pp. 9072-9084, 2018.

2. B. Wang, F.C. Zou, and Y. Zhang, "New memritive chaotic system and the application in digital watermark," Optik, vol. 172, pp. 873-878, 2018.

3. R. Lan, J. He, S. Wang, T. Gu, and X. Luo, "Integrated chaotic systems for image encryption," Signal Processing, vol. 147, pp. 133$145,2018$.

4. Y. Wang and H. Yu, "Fuzzy synchronization of chaotic systems via intermittent control," Chaos, Solitons \& Fractals, vol. 106, pp. 154-160, 2018.

5. Q. Lai, A. Akgul, M. Varan, J. Kengne, and A.T. Erguzel, "Dynamic analysis and synchronization control of an unusual chaotic system with exponential term and coexisting attractors," Chinese Journal of Physics, vol. 56, pp. 2837 2851, 2018.

6. T.L Le, C.M. Lin, and T.T. Huynh, "Self-evolving type-2 fuzzy brain emotional learning control 
design for chaotic systems using PSO," Applied Soft Computing, vol. 73, pp. 418-433, 2018.

7. R. Behinfaraz and M.A. Badamchizadeh, "Synchronization of different fractional order chaotic systems with time-varying parameter and orders," ISA Transactions, vol. 80, pp. 399-410, 2018.

8. H. Tirandaz, S.S. Aminabadi, and H. Tavakoli, "Chaos synchronization and parameter identification of a finance chaotic system with unknown parameters, a linear feedback controller," Alexandria Engineering Journal, vol. 57, pp. 1519-1524, 2018.

9. P.P. Singh and B.K. Roy, "Comparative performances of synchronisation between different classes of chaotic systems using three control techniques," Annual Reviews in Control, vol. 45, pp. 152-165, 2018.

10. H. Wang, Y. Liu, and J.H. Park, "Stabilization of chaotic systems under variable sampling and state quantized controller," Fuzzy Sets and Systems, vol. 344, pp. 129-144, 2018.

11. H. Peng, X. Wang, B. Shi, S. Zhang, and B. Chen, "Stabilizing constrained chaotic system using a symplectic psuedospectral method," Communications in Nonlinear Science and Numerical Simulation, vol. 56, pp. 77-92, 2018.

12. D. Ma, X. Li, Q. Sun, and X. Xie, "Fault tolerant synchronization of chaotic systems with time delay based on the double event-triggered sampled control," Applied Mathematics and Computation, vol. 333, pp. 20-31, 2018.

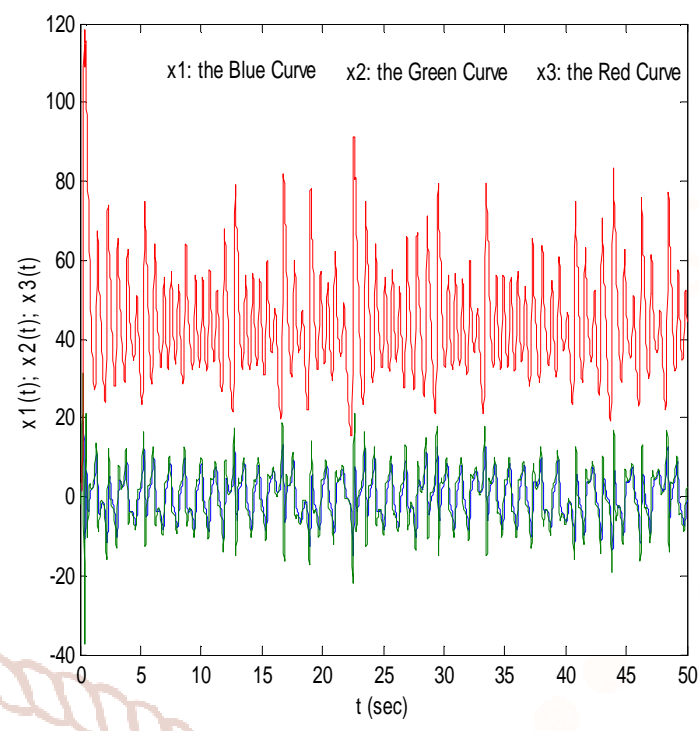

Figure 1: Typical state trajectories of the system (1) with $u=0, a_{1}=-a_{2}=10, a_{3}=40, a_{4}=-1, a_{5}=a_{6}=0$, $a_{7}=-2.5$, and $a_{8}=4$.

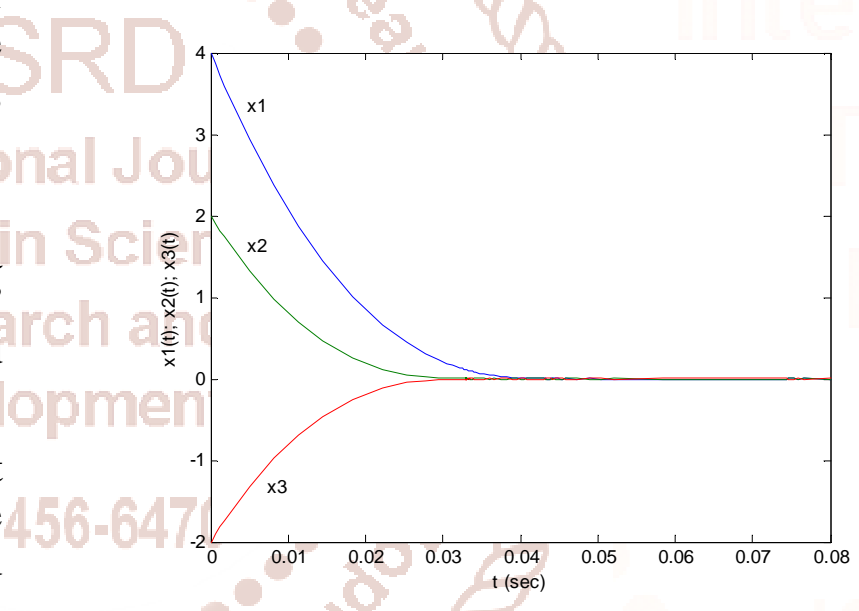

Figure 2: Typical state trajectories of the feedbackcontrolled system of (1) with (9). 